\title{
The Use of Artificial Intelligence in Traffic Violation Data Analysis
}

\author{
Mohammed Alshriem \\ ICT Division, CSE, Hamad Bin Khalifa University, Doha, Qatar.
}

ORCID: 0000-0002-0252-6554

\section{INTRODUCTION}

Artificial Intelligence, or AI, is profoundly impacting the ways in which people across the globe interact. Being a powerful set of technologies, people have been helped in solving almost every everyday problem, which makes AI's applicable in numerous fields [1]. One of which is transportation that has already been disrupted the ways in which the people and goods are moved. In addition, AI has also been playing an important role in transportation section from scanning of the traffic patterns to the reduction of the road accidents, and from the optimisation of the routes to the minimisation of the emission [2]. All of these have been made into reality through data collection and analysis; thereby indicating that AI has been critical for the creation of opportunities to make transport much safer, cleaner, efficient and reliable at the same time. In both emerging markets and advanced economies, AI's multiple applications have exemplified the contributions through evolving technologies, while effectively managing the challenges posed by these technologies [3].

In the sixth decade, AI has made substantial progress as a discipline, and more recently the advancements in AI have led to acceleration that has been supported through the machine learning evolution, in addition to the enhanced and improved computing power as well as communication networks and data storage [4]. Based on the definition put forward by EM Compass Note, AI can be explained as a series of methods, technologies, and approaches, instead of being one type of robot or machine [5], that displays the intelligent behaviour through more advanced analysis of the environment to take actions with some degree of autonomy so that the specific targets can be achieved for bringing radical improvements in the mode of transportation [4].

\section{APPLICATION OF ARTIFICIAL INTELLIGENCE (AI) IN TRANSPORT AND TRAFFIC MANAGEMENT / VIOLATION}

AI has radically and fundamentally changed the world economy, and has been predicted to continue doing so in the future. According to analysts, the capability of AI in terms of analysing the data resulting from the advancements in AI could result in the addition of $\$ 13$ trillion to the global economic output by the next decade (i.e. 2030) [6]. The forecast also takes account of the transportation sector, where the application of AI has been predicted to result in additional disruptions. During 2017, the transportation-related AI technologies in the global market reached between $\$ 1.2$ to $\$ 1.4$ billion, which is expected to grow to $\$ 3.1$ to $\$ 3.5$ billion by 2023 . If these predictions are specifically taken into account, the Compound Annual Growth Rate to be registered would be somewhere between 12 and 14.5 per cent during the time period i.e. 2017 to 2023 (see Figure 1). The rapid growth of AI can be attributed to the benefits associated with it, in specific reference to the transportation sector in the form of increased efficiency, safety of both drivers and pedestrian, lower costs, and minimised possibility of traffic violation [7].

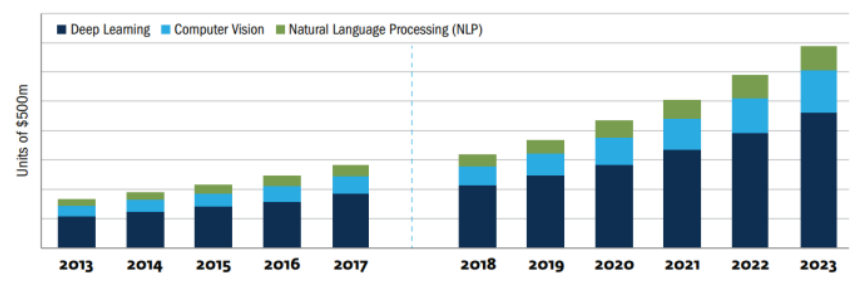

In a vast majority of the cases, it has become exceptionally difficult to fully comprehend the relationship that exists between the characteristics of the transportation system; thereby making AI one of the most lucrative methods and smart solution to analyse the data within the complex systems, which is near to impossible through the traditional methods. This is the reason that many researchers have acknowledged AI to have several benefits with respect to transportation [8]; for instance, the transformation of the data obtained via traffic sensors into smart agents for the purpose of identifying and detecting violations as well as accidents, while predicting the ways to improve future conditions [11].

With several methods integrated within AI in transportation management like Artificial Neutral Networks (ANNs), which are generally used for the purpose of road planning [12] as well as public transport [13, 14], and the detection of traffic incidents [15-17], and traffic condition predictions etc. [9] ANN can be further classified into unsupervised and supervised learning methods. The unsupervised classification ANNs include cluster analysis and greedy layer-wise; whereas the supervised methods include; Radical Basis Network (RBN), Support Vector Machine (SVM), K-Nearest Neighbours and Decision Tree, and lastly the Probabilistic Neutral Network (PNN).

Failure to analyse the transportation data can result in optimisation issues that needs bespoke algorithms for the purpose of computational analytics, which are easy to solve. This is due to these being highly advanced computational algorithms, which can be referred as raster algorithms. One of 
the most prominent example of such algorithm is the Genetic Algorithm (GA), which has been based on the evolutionary biological concept and can help in solving complex optimisation problems. The GA is based on the concept of 'survival of the fitness' that makes it a better tool to be used when designing urban networks [18-21].

Swarm Intelligence System uses both Artificial Immune System (AIS) and Bee Colony Optimisation (BCO) [22-25]. This system uses the data to represent uncertainty, in addition to the vague and imprecision concepts; thereby making it among those techniques that can be used for the purpose of route optimisation in the transportation section. Fuzzy Logic Model (FLM) has been commonly cited with respect to optimisation, and has been consistently used for the purpose of solving shortest path optimisation [25]. When compared with Logistic Regression Model (LRM), FLM outperformed it in the development of route choice model [26]. Therefore, it has been indicated that intelligent techniques mentioned earlier are more reliable and suitable for the purpose of predicting, reasoning and adaptability.

Also, Neural Networks (NNs) have been continually integrated with the systems for more improved and credible results. For instance, it has been showed that its application in the transportation sector can help the authorities in determining the ways to effectively relieve congestions, while making the travel more reliable. However, this can never be achieved without the data analysis supported through AI, which have been cited to have resulted in improved productivity and economics of the vital assets.

\subsection{Planning, Designing and Controlling Transportation Network Structure through AI}

The purpose of planning has been to work on the identification of the community needs, while deciding on the best approach or approaches through which the demands can be met without increasing the negative consequences for the environmental, economic and social aspects in transportation. Network Design Problem (NDP) has been identified to be associated with the purpose of designing optimal road method in transportation management [27]. In this context, it has been identified that there are both continuous and discrete problems associated with transportation. The continuous problem exists in the form of changes in the existing infrastructure capacity; meanwhile the discrete problem has been identified in the form of adding more infrastructure.

During the 1990s, the focus remained on the use of NNs for the purpose of planning, designing and modelling the roads. For instance, Rodrigue modelled the spatial relationship between land-use planning and transportation through the use of the parallel NN system [28]. However, the research by Li et al. focused more on the application of the raster algorithms due to their preference with respect to urban planning [29]. This was further justified by the authors in a simplistic manner that is the lack of existing nodes and links in finding the optimal path.

In the 21 st century, voluminous data are analysed through the use of advanced algorithms, which have remained the centre of attention in specific reference to machine learning. It has created opportunities in terms of creating patterns among the data, which was an impossible thing to do in the past. Xu et al. focused on addressing the continuous NDP problem through the application of GA and SA algorithms, while comparing their efficiency given the simulated network [30]. The authors found that SA can help in finding optimal value with lower focus on computational efforts when compared with GA, but this is only possible with lower demand. However, GA through its advanced computations can help in reaching optimal and better solution.

This has been opposed in the research by Karoonsoontawong and Waller, where the authors acknowledged GA given its provision of better and improved results when compared with SA due to the less computational time. However, the model used within the study was single-level linear model given the case of continuous NDP problem [31].

Safety management plan has been modelled in the research by Akgungor and Dogan for the Ankara City in Turkey. The research applied Genetic algorithm (GA) and ANN [32]. The study confirmed that the application of ANN is more suitable against GA. In particular, ANN was found to have lower/less error involved in comparison to GA. On the contrary, Wen and $\mathrm{Li}$ applied the Ant Colony Algorithm for the purpose of designing optimal vehicle path [33]; whereas $\mathrm{Li}$ et al. considered the combination of Cellular Automata (CA) spatial simulation method - in addition to Ant Colony Optimisation technique [34]. The findings of the study showed planning improvement with respect to urban development, and the pattern of land usage given the case of simulated industrial land in China.

Furthermore, Dogan and Akgungor focused specifically on the safety plan and good transportation management system so that a balance can be created with respect to the demand of transportation through proper distribution of railways, highways and even airways [35]. ANN has been used by the authors for the purpose of predicting accident and injury between the highly congested route in Turkey (i.e. from Istanbul and Ankara).

Over the years, it has been recognised that routes planning for the vehicles have become a necessity. Doing so can help in reducing and avoiding congestions a well as delays when travelling many times in a day. In this context, the ant colony algorithm has been proposed as a promising solution for the routing problem for vehicles, which has been recognised and acknowledged to be a leading problem. In this context Bell and McMullen used BCO algorithm to resolve the wavelength and routing problem [36], which means that it is impeccable to choose a path within the network as well as the assignment of the wavelength path for the connected nodes. This was argued to be critical in maximising the number of connection established, especially in between the nodes within the network.

Furthermore, the research conducted recently focused on the use of microscopic traffic data to model and identify the security breach, as well as for the purpose of traffic control system and for the roadway management plan [37]. With respect to the purpose of identifying the best and most suitable path for the users of public transport, Nuzzolo and Comi 
suggested the value of learning and updating the real time path generation system in accordance with the travellers' preferences [38]. In addition, the application of the utilitybased approach was suggested to be an effective approach because of its concentration of the different paths' attributes and parameters with respect to the case of the users of public transport.

Intelligence Transport System (ITS) is among the areas, where the applications of AI have seen rapid developments. The purpose of these systems has been to alleviate congestions, while improving the driving experience through the use of numerous communication and technologies. The AI helps in capturing critical data that can be further integrated with the machine learning technology [39]. For instance, Liu elaborated on the application of the deep reinforcement learning for real time optimisation of the policies associated with traffic control, which are embedded into the large scale ITS systems [39]. Furthermore, Ferdowsi et al. further confirmed the value of AI by suggesting that deep learning systems have empowered the ITS devices, while improving their functionalities given the cases of signal processing as well as fast computing analytics [40].

The continuous development of the ITS can result in increased data complexity in the future; thereby making it necessary to find features and patterns through deep learning techniques. Doing so, can help in ensuring more connected and advanced transportation systems. In this context, Wang et al. considered the use of fuzzy methods and genetic algorithms for the purpose of controlling traffic signal systems at different intersections. 'NeverStop' was proposed through the use of ITS system [41], which relied on the use of RFIC sensors, and the findings indicated that this was among the effective methods through which the average waiting times for the vehicles can be reduced. In addition, Gilmore developed two Neural Networks (NNs) so that the roads can be managed more efficiency through the use of microscopic simulated data [42]. The first NN system focused on controlling the traffic signal; meanwhile the second focused specifically on the prediction of the future traffic congestions.

Lastly, Fries et al. [43] and Nuzzolo and Comi [44] argued about the use of reinforcement learning so that the systems' parameters and cycle length can be updated with the change in the periodic flow. This makes AI a dynamic research area given the case of transportation management, which has presented several innovative and technologically advanced methods to improve the decision making, planning and the management of the road.

\subsubsection{Incident Decision}

Over the years, several attempts have been made for the purpose of establishing identity, time, location and severity of incident so that the traffic managers can be supported for the purpose of mitigating congestions. These attempts have been in the form of manual reports as well as automated algorithms, and Neural Networks. In particular, the manual reports are developed and written by humans, which means that there is always a possibility of delays in the detection of the incidents; thereby making them costly. On the other hand, algorithms are focused on measuring the flow characteristics pre and post incidents, where the data is collected through the sensors along the road. For incident detection, California Algorithm was first statistical technique to detect incidents; however, it has been reflected that arterial roads make it difficult to use algorithms, since there are traffic signals as well as street parking.

This might be considered one of the many reasons that Neural Networks approaches have been commonly used for the purpose of developing algorithms. Dia and Rose argued about the classification of $\mathrm{NN}$ algorithm, and its evaluation given the case of detecting incident occurrence in freeway [45]; whereas Stojmenovic elaborated on the need of finding an effective software package that can detect all vehicles' objects in realtime [46]. This is the reason that AdaBoost software was recommended to be used for the purpose of accurate image detection. On the other hand, Wang and Work proposed considering IMM ENKF algorithm so that the incidents can be detected given hybrid state problems [47]; thereby leading to the development of more advanced and accurate algorithm 'Efficient Multiple Model Particle Filter' (EMMPF) so that the incidents can be detected on highways through the use of both field and simulated data.

Real time incidents have been indicated to be detected through social media as well, and this has been discussed in the findings of $\mathrm{Gu}$ et al. In particular, the authors demonstrated its application over Twitter, which was cited to be an efficient and cost-effective techniques that can help in acknowledging the occurrence of incidents on both arterial roads and freeway [48].

\subsubsection{Predictive Models}

The need to have advanced methods for the purpose of predicting traffic information can be attributed to the rapid development in Intelligent Transport System. In this context, it has been identified that the application of these methods have a critical role in the success of the ITS subsystems like advanced traffic management system, advanced traveller information systems and commercial vehicle operations. Mahamuni [49] recognised that the ITS are developed through the use of historical data, which are extracted from the sensors attached to the roads, which are then used as an input in the AI algorithms and machine learning for short and long-term as well as realtime predictions.

Simple Feedforward Neural Network was used for the purpose of predicting flow of short-term; for instance, Ledoux worked on NNs, where one of the layers was hidden to the overall urban traffic control [50]. This helped in demonstrating the ability of strongly predicting the flow of the traffic up to 1 minute through the use of simulated data only. On the other hand, Dia considered the use of field data from 1.5 kilometre section of highway in Queensland [51]. Doing so helped the author in predicting the speed of the vehicle for 5 minutes into the future, and had accuracy percentage of 90-94. It is worth mentioning that when flow and speed are being used as an input in the network, it can help in predicting the travel time for approximately 15 minutes, which can be argued to be accurate as speed prediction. 
Another example has been presented in the study by Lv et al., where a deep NN was developed by the authors for the purpose of predicting the flow of traffic by 60 minutes into the future [52]. The data, in this context, was collected from freeways across California, and unsupervised stack of auto-encoder (SAE) model was used, which was then trained through the use of greedy layer-wise algorithm. The important features of the traffic flow were extracted as the output was fed back, as an input into the network, and the application of the supervised logistic layer further made it possible to predict.

Another AI technique that has been able to predict the flow of traffic has been presented in the study by More et al. [53] where the authors worked on the development of a simple recurrent $\mathrm{NN}$ for the purpose of short-term forecasting. This was done through the application of Jordan's NN. It was further stated that the Jordan's NN was different from the conventional feedforward network because of the context layer's addition, which was responsibility for the storage of previous information, while acting as a memory box. The already stored information ( $\mathrm{t}-1$ ) was feedforwarded to the hidden layer with the input at time $(\mathrm{t})$. Doing so helped the network in predicting the subsequence, which is the reason that it is commonly termed as Jordon's Sequential Network as well. With their NN at work, the authors were able to prove that the network that accuracy of the network can be improved by increasing the number of the neurons in the hidden layer, when compared with the number of input neurons. Also, the authors were able to identify that 0.5 was the learning rate, and that the decrease in iterations can result in improving the prediction accuracy between 92-98 per cent.

In the $21^{\text {st }}$ century, Uber as a ride-hailing service has played an instrumental role in collecting massive amount of data. In this context, Yao et al. elaborated that AI can play an instrumental role because of the data [54], which can be used not just in predicting the demands from the passengers, but also in terms of avoiding empty vehicles, which in turn can help in reducing the congestions as well as energy consumption. Furthermore, the authors proposed deep learning model using Multi-View, Spatial and Temporal Network, which can help in scaling ridesharing and ride-hailing demands, but this was more specifically based on DiDi Chuxing in China. The combination of local CNN capturing data from local regions in specific reference to their surrounding areas [56], and Long-Short Term Memory Network (LSTM) for the purpose of modelling temporal features. The authors were able to demonstrate superior performance given the application of this particular mode [57].

\subsection{Public Transportation and Artificial Intelligence}

The advancement of the technology as well as computer science has been of instrumental importance, given the fact that it has made the data collection process more approachable and reliable. Given the fact that public transportation has remained unstructured and dense; there is greater need to model the public transportation in more effective manner so that the data obtained can be optimally leveraged and taken full advantage of.

\subsubsection{Shared Mobility}

In urban cities, where sharing economy has gained momentum, and has shown signs of being promising given the mobility can help in reducing the traffic congestion and pollution, especially in the inner-city. This can be possible through the application of $\mathrm{AI}$ and its abilities of providing strategies that may help to remove the less occupant or single personal vehicle from the road. This can be acknowledged as a win-win strategy, since it would not just result in the provision of short-term socioeconomic benefits, but can also help in efficient long-term sustainable solutions. This is only possible through the adoption of AI that can help in mitigating environmental impacts as well as traffic issues.

Airbnb and Uber being among the pioneers of sharing economy, it has been identified that the momentum of it rests within the Transportation and Accommodation sectors [59]. With respect to the case of transportation sector, AI can play an instrumental role in further development of the business models through which the new-shared mobility services can emerge that may ensure sustainable transportation system, and may even help in addressing the gap to fit the demand with the supply in an effective manner [55,60]. More specifically, the attention of the users has been driven based on the possibility of sharing rides, cars and even bikes; all of these shared mobility options can be critical in addressing the major shortcoming of 'first-mile, last-mile'.

The integration of AI technology can benefit the shared mobility, more specifically in improving the experience of the customers as well as in streamlining the businesses. The provision of the personalised experience to the customers/users is only possible through shared mobility and integration of AI. For instance, Uber through the integration of AI has been able to suggest rider destination based on the ride history of the user. In addition, the introduction of the route-based pricing by Uber is dependent upon AI that helps in predicting the willingness of the riders to pay for destination, as well as given the factors of location and time of the day. Furthermore, the application of AI has helped the Uber operators in collecting data and analysing it to identify fraudulent drivers as well as strategies through which the fraudulent activities can be prevented. From this perspective, it can be argued that the future of the shared mobility has made it evident that AI can be beneficial with the driverless cars, which Inside Big Data identified to be more profitability.

\subsubsection{Buses}

Given the case of public transportation sector, the role played by buses is simply undeniable, and scholars have focused on making the bus destinations and journeys to be more reliable and safe. In this context, it has been identified that the application of Hybrid Ant Colony Algorithm can prove to be beneficial in managing the bus schedules, while enhancing the reliability and efficiency for the bus drivers [61,62]. Furthermore, it has been argued that the ANNs, in this context, are more efficient in terms of reducing the waiting time for the passengers, which can be achieved through the prediction of bus arrival time $[63,64]$. This was further confirmed through 
the study by Han et al., where iBus was introduced based on the dual mode architecture, where the processes were found to be much similar to human driver [65]. These three processes included; perception, decision making and taking actions through the use of hardware and software. This has been tested in China.

Another prominent area worth looking into is the demand responsive public transportation, which through the use of AI advancements can be made flexible. These busses can offer ondemand service, but this relies heavily on the data collection and analysis to ensure that the operations remain flexible, as well as the schedules and routes. The application of AI can help in ensuring that the services are able to offer door-to-door services, which would be much similar to the taxis, but at a fraction of the costs along with greater efficiency. Over the years, there have been several attempts to improve the operational efficiency of the conventional bus services, while ensuring that these can provide convenient services to the users. One of the most prominent example is of Optibus, which has been introduced through the use of AI-driven on-time optimisation solution [66], where the focus has remained on the reduction of delays as well as the provision of on-time services.

\section{LIMITATIONS OF AI TECHNIQUES}

Arguably, the application of AI for data collection and analysis purpose has received much attention in the $21^{\text {st }}$ century, but the methods and techniques associated with AI have drawn much criticism, especially since its association with the transportation sector. One of the major limitations identified by Olden and Jackson was in the form of AI's consideration of ANNs as 'black box' [67]. From a more critical perspective, it has been identified that the relationship between the output and input is developed with the internal computation's knowledge. At the same time, it has been identified that the ANNs has the ability of generalisation, specifically when there is a missing data or information [68-70]. However, the research studies by Kanungo et al. [68], Setiono et al. [69], and Dayhoff and DeLeo [70] focused on overcoming this particular limitation through the combination of the $\mathrm{NN}$ with traditional techniques, in addition to the Hybrid Solutions (AI tools) so that the problems can be fixed. However, the research by Harcourt recognised hybridisation for the purpose of improving the performance, especially in the multi-scenario as a general weakness of AI's implementation in the transportation sector.

Another limitation identified, over the years, has been argued to be the ability of reaching the most optimal solution with respect to the AI tools like ACO and GA [71]. In addition, it has been recognised that the mathematical computations methods can be instrumental in providing information and true understanding of the problems' internal structure as well as the solutions' nature, unlike through the application of AI raster algorithms. However, it would become impossible to consider the application of traditional mathematical techniques, especially given the case of hard optimisation problems, which means that the algorithms can generate quick analytical results are more suitable and good approach, when compared with no solution. This can be further confirmed through the findings of Chowdhury and Sadek [71], where both the authors identified the application of these algorithms in reaching good solutions in majority of the cases.

In the transportation, it is of exceptional importance to have the capabilities of forecasting both short and long-term flow of the traffic. However, the challenge identified in this context is related to the forecasting of adverse weather conditions as well as the unexpected events. Unfortunately, the focus of AI has not been on such instances, which means that there is no particular technique to tool available to do so; thereby calling on immediate attention on developing incident and weather responsive algorithms to ensure higher accuracy.

\section{FUTURE OF AI AND DEEP LEARNING}

The innovation in the form of deep learning has continuously helped in unfolding the mystery behind the ways in which voluminous data is generated in different industries. According to Grand View Research, deep learning technique had a market value of \$272 million during 2016 [72], which was forecasted to grow because of the increasing capacity of data storage, followed by more accurate computing power, along with the abilities of managing complexity within voluminous data. Though, this value was based on the deep learning application with respect to image recognition tasks in healthcare sector as well as the facial recognition feature of Facebook. However, if the automotive and financial sectors along with the data mining sector are specifically taken into consideration, it would reflect that all of these sectors have continually improved their operations through the adoption and application of deep learning AI technology. In addition, the ability to recognise the data patterns to make future predictions based on data analysis has help reflected to result in the rapid growth of deep learning by 2025 , which is expected to hit $\$ 10.2$ billion [73].

Studies, identified and analysed, in this report has helped in identifying the AI usage and the fact that the value of it can be further improved for the travel and transport sectors. It has been noted that the modern [74] AI's deep learning NN can help in the provision of better value when compared with the traditional techniques, as reflected in Figure 2;

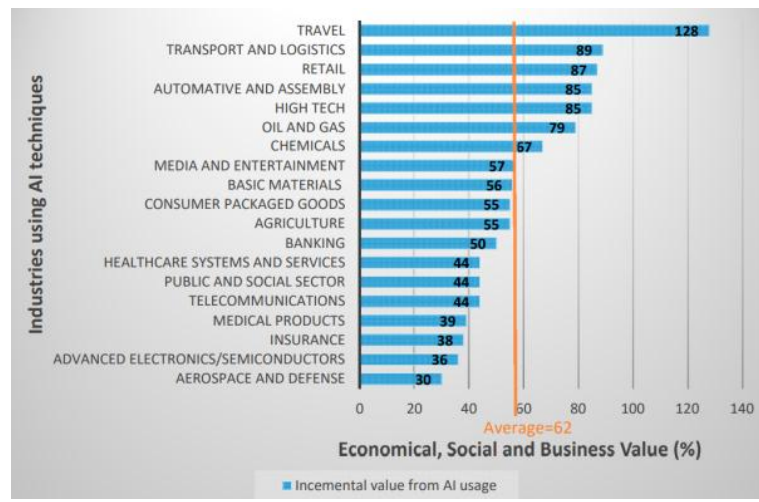

The figure reflects that there are several benefits to be derived from the travel and transport related services; for instance, the application of the AI techniques can be used for the purpose of finding reliable and accurate, as well as optimum and fastest route for the convenience of the users, while further advancing the role to the delivery services. The detection of real-time 
information can be further illustrated through the case of a European Company, which not only analysed the information from the roads' sensors, but also considered the driver behaviour. Doing so, helped the company in reducing 15 per cent of their fuel costs, as well as it resulted in the minimising the delivery time. It is worth mentioning that the application of AI is not just related to land transportation, but can also help airlines in avoiding the costs of cancellation by more effectively predicting and forecasting the weather conditions.

\section{FUTURE RESEARCH WORK}

The findings of this research has clarified about ANN being a robust model because of its ability of covering multiple tasks associated with AI, and the fact that it does not concentrate or need deep understanding of the processes. The advantage of it also includes the relationship between the inputs and outputs for the purpose of recognising the patterns, which may further help in the management of voluminous data through the welladjusted and performance when surrounding by noisy data. The fast computation tool can help in reducing the time, while improving the performance. Arguably, there are several researches that have only focused on one or two of the traffic parameters for the purpose of developing the model, which means that the future researchers can focus on enhancing the predictive operations through the use of more than two features. In addition, the future researchers can further work on more than one hidden layer for the model's structure.

\section{CONCLUSION}

Conclusively, this research has presented an overview of the AI's application in a variety of transport related issues. The application of AI has been proven to be critical in increasing and improving the transportation system, which can become more instrumented towards the provision of much-need data for the development of application of AI. This research has focused on the core application areas, which the researcher believed and found to be more critical in terms of their influence on the public transportation, and more broadly the transportation system.

\section{REFERENCES}

[1] Sadek, A.W., 2007. Artificial intelligence applications in transportation. Artificial Intelligence in Transportation: Information for Application, pp.1-6.

[2] Qi, L., 2008, August. Research on intelligent transportation system technologies and applications. In 2008 Workshop on Power Electronics and Intelligent Transportation System (pp. 529-531). IEEE.

[3] Sumalee, A. and Ho, H.W., 2018. Smarter and more connected: Future intelligent transportation system. IATSS Research, 42(2), pp.67-71.

[4] Niestadt, M., Debyser, A., Scordamaglia, D. and Pape, M., 2019. Artificial intelligence in transport Current and future developments, opportunities and challenges. European Parliamentary Research Service.

[5] Strusani, D. and Houngbonon, G.V., 2019. The Role of Artificial Intelligence in Supporting Development in Emerging Markets.

[6] Bughin, J., Seong, J., Manyika, J., Chui, M. and Joshi, R., 2018. Notes from the AI frontier: Modeling the impact of AI on the world economy. McKinsey Global Institute.

[7] Zheng, X., Chen, W., Wang, P., Shen, D., Chen, S., Wang, X., Zhang, Q. and Yang, L., 2015. Big data for social transportation. IEEE Transactions on Intelligent Transportation Systems, 17(3), pp.620-630.

[8] Sładkowski, A. and Pamuła, W. eds., 2016. Intelligent transportation systems-problems and perspectives (Vol. 303). Springer International Publishing.

[9] Kumar, K., Parida, M. and Katiyar, V.K., 2015. Short term traffic flow prediction in heterogeneous condition using artificial neural network. Transport, 30(4), pp.397405.

[10] Ma, X., Dai, Z., He, Z., Ma, J., Wang, Y. and Wang, Y., 2017. Learning traffic as images: a deep convolutional neural network for large-scale transportation network speed prediction. Sensors, 17(4), p.818.

[11] Bazzan, A.L. and Klügl, F., 2014. A review on agentbased technology for traffic and transportation. The Knowledge Engineering Review, 29(3), pp.375-403.

[12] Doğan, E. and Akgüngör, A.P., 2013. Forecasting highway casualties under the effect of railway development policy in Turkey using artificial neural networks. Neural computing and applications, 22(5), pp.869-877.

[13] Budalakoti, S., Srivastava, A.N. and Otey, M.E., 2008. Anomaly detection and diagnosis algorithms for discrete symbol sequences with applications to airline safety. IEEE Transactions on Systems, Man, and Cybernetics, Part C (Applications and Reviews), 39(1), pp.101-113.

[14] Akgüngör, A.P. and Doğan, E., 2009. An artificial intelligent approach to traffic accident estimation: Model development and application. Transport, 24(2), pp.135142.

[15] Dia, H. and Rose, G., 1997. Development and evaluation of neural network freeway incident detection models using field data. Transportation Research Part $C$ : Emerging Technologies, 5(5), pp.313-331.

[16] Wang, R., Fan, S. and Work, D.B., 2016. Efficient multiple model particle filtering for joint traffic state estimation and incident detection. Transportation Research Part C: Emerging Technologies, 71, pp.521537.

[17] Wang, R. and Work, D.B., 2014, October. Interactive multiple model ensemble Kalman filter for traffic estimation and incident detection. In 17th International IEEE Conference on Intelligent Transportation Systems 
International Journal of Engineering Research and Technology. ISSN 0974-3154 Vol.13, No.4 (2020), pp. 644-652

(C) International Research Publication House. https://dx.doi.org/10.37624/IJERT/13.4.2020.644-652

(ITSC) (pp. 804-809). IEEE.

[18] Xu, T., Wei, H. and Hu, G., 2009. Study on continuous network design problem using simulated annealing and genetic algorithm. Expert Systems with Applications, 36(2), pp.1322-1328.

[19] Ceylan, H. and Bell, M.G., 2004. Traffic signal timing optimisation based on genetic algorithm approach, including drivers' routing. Transportation Research Part B: Methodological, 38(4), pp.329-342.

[20] Ulusoy, G., Sivrikaya-Şerifoğ lu, F. and Bilge, Ü., 1997. A genetic algorithm approach to the simultaneous scheduling of machines and automated guided vehicles. Computers \& Operations Research, 24(4), pp.335-351.

[21] Karoonsoontawong, A. and Waller, S.T., 2006. Dynamic continuous network design problem: linear bilevel programming and metaheuristic approaches. Transportation Research Record, 1964(1), pp.104-117.

[22] Dorigo, M., Gambardella, L.M., Birattari, M., Martinoli, A., Poli, R. and Stützle, T. eds., 2006. Ant Colony Optimization and Swarm Intelligence: 5th International Workshop, ANTS 2006, Brussels, Belgium, September 47, 2006, Proceedings (Vol. 4150). Springer.

[23] Timmis, J., Neal, M. and Hunt, J., 2000. An artificial immune system for data analysis. Biosystems, 55(1-3), pp.143-150.

[24] Dasgupta, D., Ji, Z. and Gonzalez, F., 2003, December. Artificial immune system (AIS) research in the last five years. In The 2003 Congress on Evolutionary Computation, 2003. CEC'03. (Vol. 1, pp. 123-130). IEEE.

[25] Qureshi, M.F., Shah, S.A. and Al-Matroushi, G.I.G., 2013. A Comparative Analysis of Multi-Criteria Road Network. Eur. Cent. Res. Train. Dev. UK, pp.27-47.

[26] Murat, Y.S. and Uludag, N., 2008. Route choice modelling in urban transportation networks using fuzzy logic and logistic regression methods.

[27] Bagloee, S.A., Sarvi, M. and Patriksson, M., 2017. A hybrid branch-and-bound and benders decomposition algorithm for the network design problem. ComputerAided Civil and Infrastructure Engineering, 32(4), pp.319-343.

[28] Rodrigue, J.P., 1997. Parallel modelling and Neural Networks: an overview for transportation/land use systems. Transportation Research Part C: Emerging Technologies, 5(5), pp.259-271.

[29] Li, X., Shi, X., He, J. and Liu, X., 2011. Coupling simulation and optimization to solve planning problems in a fast-developing area. Annals of the Association of American Geographers, 101(5), pp.1032-1048.

[30] Xu, T., Wei, H. and Hu, G., 2009. Study on continuous network design problem using simulated annealing and genetic algorithm. Expert Systems with Applications, 36(2), pp.1322-1328.

[31] Karoonsoontawong, A. and Waller, S.T., 2006. Dynamic continuous network design problem: linear bilevel programming and metaheuristic approaches. Transportation Research Record, 1964(1), pp.104-117.

[32] Akgüngör, A.P. and Doğan, E., 2009. An artificial intelligent approach to traffic accident estimation: Model development and application. Transport, 24(2), pp.135142 .

[33] Wen, S.Y. and Li, Y., 2011. Fastest Complete Vehicle Routing Problem Using Learning Multiple Ant Colony Algorithm. In Advanced Materials Research (Vol. 217, pp. 1044-1049). Trans Tech Publications Ltd.

[34] Li, X., Shi, X., He, J. and Liu, X., 2011. Coupling simulation and optimization to solve planning problems in a fast-developing area. Annals of the Association of American Geographers, 101(5), pp.1032-1048.

[35] Doğan, E. and Akgüngör, A.P., 2013. Forecasting highway casualties under the effect of railway development policy in Turkey using artificial neural networks. Neural computing and applications, 22(5), pp.869-877.

[36] Bell, J.E. and McMullen, P.R., 2004. Ant colony optimization techniques for the vehicle routing problem. Advanced engineering informatics, 18(1), pp.41-48.

[37] Fries, R., Chowdhury, M. and Brummond, J., 2008. Transportation infrastructure security utilizing intelligent transportation systems. John Wiley \& Sons.

[38] Nuzzolo, A. and Comi, A., 2016. Advanced public transport and intelligent transport systems: new modelling challenges. Transportmetrica A: Transport Science, 12(8), pp.674-699.

[39] Abduljabbar, R., Dia, H., Liyanage, S. and Bagloee, S.A., 2019. Applications of artificial intelligence in transport: An overview. Sustainability, 11(1), p.189.

[40] Ferdowsi, A., Challita, U. and Saad, W., 2019. Deep learning for reliable mobile edge analytics in intelligent transportation systems: An overview. ieee vehicular technology magazine, 14(1), pp.62-70.

[41] Wang, C., Li, X., Zhou, X., Wang, A. and Nedjah, N., 2016. Soft computing in big data intelligent transportation systems. Applied Soft Computing, 38, pp.1099-1108.

[42] Gilmore, J.F. and Abe, N., 1995. Neural network models for traffic control and congestion prediction. Journal of Intelligent Transportation Systems, 2(3), pp.231-252.

[43] Fries, R.; Chowdhury, M.; Brummond, J. Transportation Infrastructure Security Utilizing Intelligent Transportation Systems; John Wiley \& Sons: Hoboken, NJ, USA, 2008

[44] Nuzzolo, A.; Comi, A. Advanced public transport and 
International Journal of Engineering Research and Technology. ISSN 0974-3154 Vol.13, No.4 (2020), pp. 644-652

(C) International Research Publication House. https://dx.doi.org/10.37624/IJERT/13.4.2020.644-652

intelligent transport systems: New modelling challenges. Transp. A Transp. Sci. 2016, 12, 674-699

[45] Dia, H. and Rose, G., 1997. Development and evaluation of neural network freeway incident detection models using field data. Transportation Research Part C: Emerging Technologies, 5(5), pp.313-331.

[46] Stojmenovic, M., 2006. Real time machine learning based car detection in images with fast training. Machine Vision and Applications, 17(3), pp.163-172.

[47] Wang, R. and Work, D.B., 2014, October. Interactive multiple model ensemble Kalman filter for traffic estimation and incident detection. In 17th International IEEE Conference on Intelligent Transportation Systems (ITSC) (pp. 804-809). IEEE.

[48] Gu, Y., Qian, Z.S. and Chen, F., 2016. From Twitter to detector: Real-time traffic incident detection using social media data. Transportation research part $C$ : emerging technologies, 67, pp.321-342.

[49] Mahamuni, A., 2018. Internet of things, machine learning, and artificial intelligence in the modern supply chain and transportation. Defense Transportation Journal, 74(1), pp.14-17.

[50] Ledoux, C. An urban traffic flow model integrating neural networks. Transp. Res. Part C Emerg. Technol. 1997, 5, 287-300

[51] Dia, H., 2001. An object-oriented neural network approach to short-term traffic forecasting. European Journal of Operational Research, 131(2), pp.253-261.

[52] Lv, Y., Duan, Y., Kang, W., Li, Z. and Wang, F.Y., 2014. Traffic flow prediction with big data: a deep learning approach. IEEE Transactions on Intelligent Transportation Systems, 16(2), pp.865-873.

[53] More, R., Mugal, A., Rajgure, S., Adhao, R.B. and Pachghare, V.K., 2016, December. Road traffic prediction and congestion control using artificial neural networks. In 2016 International Conference on Computing, Analytics and Security Trends (CAST) (pp. 52-57). IEEE.

[54] Geissinger, A., Laurell, C. and Sandström, C., 2018. Digital Disruption beyond Uber and Airbnb-Tracking the long tail of the sharing economy. Technological Forecasting and Social Change, p.119323.

[55] Firnkorn, J. and Müller, M., 2011. What will be the environmental effects of new free-floating car-sharing systems? The case of car2go in Ulm. Ecological economics, 70(8), pp.1519-1528.

[56] Yao, H., Wu, F., Ke, J., Tang, X., Jia, Y., Lu, S., Gong, P., Ye, J. and Li, Z., 2018, April. Deep multi-view spatialtemporal network for taxi demand prediction. In ThirtySecond AAAI Conference on Artificial Intelligence.

[57] Yao, H., Wu, F., Ke, J., Tang, X., Jia, Y., Lu, S., Gong, P., Ye, J. and Li, Z., 2018, April. Deep multi-view spatialtemporal network for taxi demand prediction. In Thirty-
Second AAAI Conference on Artificial Intelligence.

[58] Ma, J., Song, C., Ceder, A.A., Liu, T. and Guan, W. 2017. Fairness in optimizing bus-crew scheduling process. PloS one, 12(11).

[59] Geissinger, A., Laurell, C. and Sandström, C., 2018. Digital Disruption beyond Uber and Airbnb-Tracking the long tail of the sharing economy. Technological Forecasting and Social Change, p.119323.

[60] Firnkorn, J. and Müller, M., 2011. What will be the environmental effects of new free-floating car-sharing systems? The case of car2go in Ulm. Ecological economics, 70(8), pp.1519-1528.

[61] Ma, J., Song, C., Ceder, A.A., Liu, T. and Guan, W., 2017. Fairness in optimizing bus-crew scheduling process. PloS one, 12(11).

[62] Hu, J., 2001, September. Study on the optimization methods of transit network based on ant algorithm. In IVEC2001. Proceedings of the IEEE International Vehicle Electronics Conference 2001. IVEC 2001 (Cat. No. 01EX522) (pp. 215-219). IEEE.

[63] Chien, S.I.J., Ding, Y. and Wei, C., 2002. Dynamic bus arrival time prediction with artificial neural networks. Journal of Transportation Engineering, 128(5), pp.429-438.

[64] Jeong, R. and Rilett, R., 2004, October. Bus arrival time prediction using artificial neural network model. In Proceedings. The 7th International IEEE Conference on Intelligent Transportation Systems (IEEE Cat. No. 04TH8749) (pp. 988-993). IEEE.

[65] Han, W., Zhang, X., Yin, J., Li, Y. and Li, D., 2017. Architecture of iBus: A Self-Driving Bus for Public Roads (No. 2017-01-0067). SAE Technical Paper.

[66] Rosin, J., Optibus Uses Artificial Intelligence to Improve Mass Transit's On-Time Performance and Prevent Delays. 2018.

[67] Olden, J.D. and Jackson, D.A., 2002. Illuminating the "black box": a randomization approach for understanding variable contributions in artificial neural networks. Ecological modelling, 154(1-2), pp.135-150.

[68] Kanungo, D.P., Arora, M.K., Sarkar, S. and Gupta, R.P., 2006. A comparative study of conventional, ANN black box, fuzzy and combined neural and fuzzy weighting procedures for landslide susceptibility zonation in Darjeeling Himalayas. Engineering Geology, 85(3-4), pp.347-366.

[69] Setiono, R., Leow, W.K. and Thong, J., 2000. Opening the neural network black box: an algorithm for extracting rules from function approximating artificial neural networks. ICIS 2000 Proceedings, p.17.

[70] Dayhoff, J.E. and DeLeo, J.M., 2001. Artificial neural networks: opening the black box. Cancer: Interdisciplinary International Journal of the American Cancer Society, 91(S8), pp.1615-1635. 
International Journal of Engineering Research and Technology. ISSN 0974-3154 Vol.13, No.4 (2020), pp. 644-652

(C) International Research Publication House. https://dx.doi.org/10.37624/IJERT/13.4.2020.644-652

[71] Chowdhury, M. and Sadek, A.W., 2012. Advantages and limitations of artificial intelligence. Artificial Intelligence Applications to Critical Transportation Issues, 6, pp.6-8.

[72] Schmidhuber, J., 2015. Deep learning in neural networks: An overview. Neural networks, 61, pp.85-117.

[73] Moreira-Matias, L., Mendes-Moreira, J., de Sousa, J.F. and Gama, J., 2015. Improving mass transit operations by using AVL-based systems: A survey. IEEE Transactions on Intelligent Transportation Systems, 16(4), pp.16361653.

[74] Chui, M., Manyika, J., Miremadi, M., Henke, N., Chung, R., Nel, P. and Malhotra, S., 2018. Notes from the AI frontier: Insights from hundreds of use cases. McKinsey Global Institute. 